\title{
Supercritical blowup in coupled parity-time-symmetric nonlinear Schrödinger equations
}

\author{
João-Paulo Dias ${ }^{1}$, Mário Figueira ${ }^{1}$, Vladimir V. Konotop ${ }^{2,3}$, and Dmitry A. Zezyulin ${ }^{2}$ \\ ${ }^{1}$ CMAF/UL, Faculdade de Ciências, Universidade de Lisboa, \\ Avenida Professor Gama Pinto 2, Lisboa 1649-003, Portugal \\ ${ }^{2}$ Centro de Física Teórica e Computacional, Faculdade de Ciências, Universidade de Lisboa, \\ Avenida Professor Gama Pinto 2, Lisboa 1649-003, Portugal \\ ${ }^{3}$ Departamento de Física, Faculdade de Ciências, Universidade de Lisboa, \\ Campo Grande, Ed. C8, Lisboa 1749-016, Portugal
}

January 5, 2018

\begin{abstract}
We prove finite time supercritical blowup in a parity-time-symmetric system of the two coupled nonlinear Schrödinger (NLS) equations. One of the equations contains gain and the other one contains dissipation such that strengths of the gain and dissipation are equal. We address two cases: in the first model all nonlinear coefficients (i.e. the ones describing selfaction and non-linear coupling) correspond to attractive (focusing) nonlinearities, and in the second case the NLS equation with gain has attractive nonlinearity while the NLS equation with dissipation has repulsive (defocusing) nonlinearity and the nonlinear coupling is repulsive, as well. The proofs are based on the virial technique arguments. Several particular cases are also illustrated numerically.
\end{abstract}

Keywords: blowup, parity-time symmetry, nonlinear Schrödinger equation.

\section{Introduction and preliminary observations}

In this paper we consider the Cauchy problem for two coupled nonlinear Schrödinger (NLS) equations

$$
\begin{aligned}
& i u_{t}=-\Delta u+i \gamma u+\kappa v-\left(g_{1}|u|^{2}+g|v|^{2}\right) u, \\
& i v_{t}=-\Delta v-i \gamma v+\kappa u-\left(g|u|^{2}+g_{2}|v|^{2}\right) v,
\end{aligned}
$$

where $x \in \mathbb{R}^{N}, t \geq 0, \Delta$ is the $N$-dimensional Laplacian, and

$$
\begin{gathered}
u(x, 0)=u_{0}(x), \quad v(x, 0)=v_{0}(x), \quad u_{0}, v_{0} \in H^{1}\left(\mathbb{R}^{N}\right), \\
|x| u_{0}(x),|x| v_{0}(x) \in L^{2}\left(\mathbb{R}^{N}\right) .
\end{gathered}
$$

We consider $g_{1}, g_{2}, g \in \mathbb{R}$, and assume that $\gamma>0$ and $\kappa>0$ [the cases $\gamma<0$ and (or) $\kappa<0$ can be considered analogously after simple redefinition of the functions $u(x, t)$ and $v(x, t)]$. Our 
main interest is in establishing sufficient conditions for the initial conditions $u_{0}(x)$ and $v_{0}(x)$ to blow up in finite time.

The system (1a $)-(1 \mathrm{~b})$ is referred to as parity-time $(\mathcal{P} \mathcal{T})$ symmetric because of its linear counterpart $\left(g=g_{1}=g_{2}=0\right)$, which under certain conditions allows for stable propagating of the linear waves, which justifies the particular relevance of the model. The concept of $\mathcal{P} \mathcal{T}$ symmetry can be formulated also in the nonlinear case referring to the formal property as follows: if functions $u(x, t)$ and $v(x, t)$ solve the pair of equations in (1a)-(1b), then the new functions $u_{\mathcal{P} \mathcal{T}}(x, t):=\bar{v}(x,-t)$ and $v_{\mathcal{P} \mathcal{T}}(x, t):=\bar{u}(x,-t)$ also solve the same equations, provided that $g_{1}=g_{2}$ (hereafter an overbar stands for the complex conjugation).

The concept of $\mathcal{P} \mathcal{T}$ symmetry was originally employed in quantum mechanics to construct nonHermitian potentials with pure real spectra [1, 2]. Soon after that, the ideas of $\mathcal{P} \mathcal{T}$ symmetry were transferred to the optics [3]. Using the mathematical analogy between the Schrödinger equations in quantum mechanics and paraxial approximation in optics, it was shown that a medium with mutually balanced gain and losses allows for stable propagation of linear waves. Further, it was suggested in [4] that a simple implementation of $\mathcal{P} \mathcal{T}$ symmetry in optical systems can be achieved using two waveguides. This geometry was proven to be very suitable for experimental observation of the effects related to the $\mathcal{P} \mathcal{T}$ symmetry [5, 6]. Optical applications which naturally admit inclusion of the nonlinearity have stimulated further studies of the nonlinear $\mathcal{P} \mathcal{T}$-symmetric system [7]. In the two-waveguide geometry described by the coupled NLS equations with gain and dissipation, there have been considered bright [8, 9] and dark [10] solitons, breathers [11], and rogue waves [12].

Interplay between the $\mathcal{P} \mathcal{T}$ symmetry and nonlinearity was also considered in the context of Bose-Einstein condensates (BECs) [13, 14], where the coupled NLS (alias Gross-Pitaevskii) equations appear as a natural model for two BECs split in an external double-well trap. Alternatively, the $\mathcal{P} \mathcal{T}$ symmetry in BECs can be implemented using spinor components of a single atomic specie in two different ground states, one of which is pumped by an external laser and another one has (either natural or induced) loss of atoms [15. Since a three-dimensional realization of a BEC appears in the most of experimental settings, it is of interest to consider dynamics of two coupled three-dimensional, and more generally $N$-dimensional, NLS equations (11) one of which accounts for gain [equation (1a)] and the other one has dissipation [equation (1b)].

Turning now to the blowup phenomenon, in coupled conservative NLS equations it was previously discussed in Refs. [16, 17] without linear coupling and in Ref. [18] with linear coupling (see also [19] and references there in for the critical collapse). On the other hand, blowup in a single NLS equation with homogeneous and inhomogeneous linear damping was considered in [20, 21] and 22, respectively.

As mentioned above, particular interest in analyzing the blowup in the $\mathcal{P} \mathcal{T}$-symmetric coupled NLS equations (1a)-(1b) stems from the fact that depending on the relation among parameters the system can display features either of a conservative medium, where linear waves [i.e. solutions of the Eqs. (11) with $g_{1}=g_{2}=g=0$ ] can propagate stably or of a dissipative one where a linear mode is unstable (i.e. it either decays or infinitely grows with time). The former case is referred to as the unbroken $\mathcal{P} \mathcal{T}$-symmetric phase [1], and corresponds to

$$
\kappa>\gamma>0 \text {. }
$$

If $\gamma>\kappa>0$, then the $\mathcal{P} \mathcal{T}$ symmetry is said to be broken. The particular case $\kappa=\gamma$ corresponds to the exceptional point [23] of the underlying linear operator. 
Thanks to the possibility of stable propagation of linear modes, the case of unbroken phase is of special physical interest. It also allows one to make some general preliminary conclusions about blowing up and global existence of solutions. Indeed, if (2) is satisfied one can perform the transformation

$$
\left(\begin{array}{l}
u \\
v
\end{array}\right)=\left(\begin{array}{cc}
e^{i \alpha / 2} & -e^{-i \alpha / 2} \\
e^{-i \alpha / 2} & e^{i \alpha / 2}
\end{array}\right)\left(\begin{array}{c}
U \\
V
\end{array}\right)
$$

where the constant phase $\alpha$ is determined by the relation

$$
e^{i \alpha}=-\frac{\kappa}{\sqrt{\kappa^{2}-\gamma^{2}}-i \gamma} .
$$

We notice that (2) implies that $\alpha \in[0, \pi / 2)$. The newly introduced functions $U(t)$ and $V(t)$ solve the system

$$
i U_{t}=-\Delta U+\omega U-F_{1}, \quad i V_{t}=-\Delta V-\omega V-F_{2}
$$

with $\omega=\sqrt{\kappa^{2}-\gamma^{2}}$,

$$
\begin{gathered}
F_{1}=G|U|^{2} U+G_{+}|V|^{2} U+2 Q|U|^{2} V+M|V|^{2} V+P U^{2} \bar{V}+(G-2 g) V^{2} \bar{U}, \\
F_{2}=\bar{G}|V|^{2} V+G_{+}|U|^{2} V+2 \bar{Q}|V|^{2} U+M|U|^{2} U+\bar{P} V^{2} \bar{U}+(\bar{G}-2 g) U^{2} \bar{V},
\end{gathered}
$$

and the other parameters being defined by

$$
\begin{array}{r}
G_{ \pm}=\frac{1}{2}\left(g_{1} \pm g_{2}\right), \quad G=g+G_{+}-i G_{-} \tan \alpha, \\
M=-\frac{G_{-}}{\cos \alpha}, \quad Q=-M+i g \sin \alpha, \quad P=M \cos (2 \alpha)-2 i G_{+} \sin \alpha
\end{array}
$$

and the initial conditions

$$
U(x, 0)=U_{0}(x), \quad V(x, 0)=V_{0}(x), \quad U_{0}, V_{0} \in H^{1}\left(\mathbb{R}^{N}\right) .
$$

Notice that the performed transformation (3) does not depend on the dimensionality of the space $N$.

If $g_{1}=g_{2}$ we have $G_{-}=M=0$ and the corresponding system (5) has particular solutions which can be found in the form $V \equiv 0$ with $U$ solving the equation

$$
i U_{t}=-\Delta U+\omega U-\left(g+g_{1}\right)|U|^{2} U .
$$

(analogously one can consider a particular solution with $U \equiv 0$ ). This leads to a number of conclusions (which follow from the well known results on the NLS equation, see. e.g. [24]) as follows.

First, if $g+g_{1}>0$ and $N \geq 2$, then there exist solutions blowing up in finite time. This collapse is characterized by the conserved squared norms $\|u\|_{2}^{2}=\|v\|_{2}^{2}=\|U\|_{2}^{2}$ and simultaneous blowup of both the fields $u$ and $v$. Notice that hereafter we use the abbreviated notation for the standard $L^{n}$-norm: $\|\cdot\|_{n}=\|\cdot\|_{L^{n}}$.

Second, if $g+g_{1}<0$ then there exist global (dispersive) solutions.

Third, subject to sufficiently small initial conditions and $N=2$ (the critical case) one can find solutions existing globally. Moreover, in the case $g_{1}=g_{2}=-g$ a solution with either $V \equiv 0$ or $U \equiv 0$ with smooth localized initial data exists globally, since it is described by the linear Schrödinger equation.

Below in this paper we concentrate on $N \geq 3$ corresponding to the super-critical case. 


\section{Main results}

In order to formulate our main results it is convenient to define the following integral Stokes components for the NLS equation:

$$
\begin{aligned}
& S_{0}=\|u\|_{2}^{2}+\|v\|_{2}^{2}, \quad S_{1}=2 \operatorname{Re} \int u \bar{v} d x, \\
& S_{2}=2 \operatorname{Im} \int u \bar{v} d x, \quad S_{3}=\|u\|_{2}^{2}-\|v\|_{2}^{2} .
\end{aligned}
$$

Hereafter for the sake of brevity we use the notation $\int \ldots d x=\int_{\mathbb{R}^{N}} \ldots d x$. Obviously, $S_{0}$ is a conserved quantity for $\gamma=0$, but becomes time-dependent for $\gamma>0$. Indeed, it is straightforward to compute

$$
\frac{1}{2} \frac{d S_{0}(t)}{d t}=\gamma S_{3}(t)
$$

which readily gives the estimate

$$
S_{0}(t) \leq S_{0}(0) e^{2 \gamma t}
$$

We also define the energy

$$
E(t)=\int\left(|\nabla u|^{2}+|\nabla v|^{2}+\kappa(u \bar{v}+\bar{u} v)-\frac{g_{1}}{2}|u|^{4}-\frac{g_{2}}{2}|v|^{4}--g|u|^{2}|v|^{2}\right) d x
$$

which is another conserved quantity in the conservative case $(\gamma=0)$. For arbitrary $\gamma$ the energy changes with time according to

$$
\frac{d E}{d t}=2 \gamma \int\left(|\nabla u|^{2}-|\nabla v|^{2}-g_{1}|u|^{4}+g_{2}|v|^{4}\right) d x
$$

Next, we define the mean squared width of the solution $X$

$$
X(t)=\int|x|^{2}\left(|u|^{2}+|v|^{2}\right) d x
$$

and its derivative $Y$ :

$$
Y(t) \equiv \frac{d X(t)}{d t}=4 \operatorname{Im} \int(u x \cdot \nabla \bar{u}+v x \cdot \nabla \bar{v}) d x+2 \gamma \int|x|^{2}\left(|u|^{2}-|v|^{2}\right) d x
$$

For the second derivative of $X(t)$ one can compute

$$
\begin{array}{r}
\frac{d^{2} X(t)}{d t^{2}}=4 N E(t)+4 \int(2-N)\left(|\nabla u|^{2}+|\nabla v|^{2}\right) d x+4 \gamma^{2} X+ \\
+16 \gamma \operatorname{Im} \int(\bar{u} x \cdot \nabla u-\bar{v} x \cdot \nabla v) d x+8 \gamma \kappa \operatorname{Im} \int|x|^{2} v \bar{u} d x- \\
-8 \kappa N \operatorname{Re} \int u \bar{v} d x .
\end{array}
$$


Now we introduce functions

$$
\begin{aligned}
F(t) & =X(0)+Y(0) t+\frac{8 N}{N+2} E(0) t^{2}+\frac{4 \kappa}{\gamma^{2}} S_{0}(0)\left(e^{2 \gamma t}-2 \gamma t-1\right), \\
M(t) & =\sup _{\tau \in[0, t]} F(\tau)+1>0 \\
G(t) & =M(t)\left(c_{1} \frac{t^{2}}{2}+\exp \left(\frac{c_{3} \gamma t}{c_{2}}\right)-1\right)
\end{aligned}
$$

where $S_{0}(0)=\left\|u_{0}\right\|_{2}^{2}+\left\|v_{0}\right\|_{2}^{2}$ is defined by $L^{2}\left(\mathbb{R}^{N}\right)$-norms of the initial conditions $u_{0}$ and $v_{0}$, as well as constants

$$
\begin{aligned}
& c_{1}=4 \gamma \kappa+4 \gamma^{2} \frac{5 N+6}{N-2}, \\
& c_{2}=\left\{\begin{array}{cl}
\frac{4}{5} \min \left\{1, g_{1}, g_{2}\right\}, & \text { if } g \geq 0, \\
\frac{4}{5} \min \left\{1, g_{1}+\frac{g \sqrt{g_{1}}}{\sqrt{g_{2}}}, g_{2}+\frac{g \sqrt{g_{2}}}{\sqrt{g_{1}}}\right\}, & \text { if }-\sqrt{g_{1} g_{2}}<g<0,
\end{array}\right. \\
& c_{3}=\frac{32 N}{N+2} \max \left\{1, g_{1}, g_{2}\right\}
\end{aligned}
$$

Our main result consists in the following Theorem which provides sufficient conditions for the finite-time blowup of solution for the problem (1) in the supercritical case.

Theorem 1 Let $N \geq 3$ and

$$
g_{1,2}>0, \quad g>-\sqrt{g_{1} g_{2}} .
$$

Assume that the initial conditions $u_{0}(x)$ and $v_{0}(x)$ of the Cauchy problem (1) are chosen such that there exists $T_{0}>0$ for which the following two conditions hold:

$$
\begin{array}{r}
F\left(T_{0}\right)+1<0, \\
G\left(T_{0}\right)<1 .
\end{array}
$$

Then the solution of the problem (1) does not exist in the interval $t \in\left[0, T_{0}\right]$.

Before presenting the proof of the Theorem 1, it is useful to ensure that the conditions (18) and (19) are consistent and that the initial conditions $u_{0}$ and $v_{0}$ satisfying (18)-(19) do exist. We illustrate the consistency of the conditions (18) and (19) formulating the two lemmas as follows. Lemma 1 establishes that the blow up occurs if the initial energy is negative (with large enough absolute value), while Lemma 2 shows that the blow up conditions can be satisfied if $Y(0)$ is negative (with large enough absolute value).

Lemma 1 Let $\beta=\frac{c_{3} \gamma}{c_{2}}$,

$$
C_{0}=\frac{|Y(0)|}{2 \gamma}+\frac{4 \kappa}{\gamma^{2}} S_{0}(0), \quad \tilde{M}(t)=1+X(0)+C_{0}\left(e^{2 \gamma t}-1\right)
$$


and

$$
\begin{aligned}
& T_{0, \text { max }}=\frac{1}{\beta} \ln \left(1+\frac{\beta^{2}}{(1+X(0))\left(\beta^{2}+c_{1}\right)}\right), \\
& T_{0, \text { min }}=\frac{1}{\beta} \ln \left(1+\frac{\beta^{2}}{\tilde{M}\left(T_{0, \text { max }}\right)\left(\beta^{2}+c_{1}\right)}\right) .
\end{aligned}
$$

Then there exists $T_{0} \in\left[T_{0, \text { min }}, T_{0, \text { max }}\right]$ such that the blow-up conditions (18)-(19) are satisfied at $t=T_{0}$ provided that

$$
E(0)<-\frac{(N+2) \tilde{M}\left(T_{0, \max }\right)}{8 N T_{0, \min }^{2}} .
$$

Proof. Let us introduce

$$
\tilde{G}(t):=M(t)\left(1+\frac{c_{1}}{\beta^{2}}\right)\left(e^{\beta t}-1\right)>G(t) \text { for } t>0,
$$

and define $T_{0}$ as the smallest solution of the equation $\tilde{G}\left(T_{0}\right)=1$ (which implies that $G\left(T_{0}\right)<1$ ). Using that $M(t) \geq 1+X(0)$ we find that $T_{0} \leq T_{0, \max }$. Introduce $\tilde{F}(t)=F(t)-\frac{8 N}{N+2} E(0) t^{2}$. For $E(0) \leq 0$ we have $\tilde{F} \geq F(t)$. With simple transformations we obtain that $\tilde{F}(t)+1 \leq \tilde{M}(t)$ and therefore for all $t \in\left[0, T_{0, \max }\right]$ one has

$$
M(t) \leq \sup _{\tau \in[0, t]} \tilde{F}(\tau)+1 \leq \sup _{\tau \in[0, t]} \tilde{M}(\tau)=\tilde{M}\left(T_{0, \max }\right),
$$

which implies that $T_{0} \geq T_{0, \text { min }}$.

In order to satisfy the condition (18) we require $0>F\left(T_{0}\right)+1=\tilde{F}\left(T_{0}\right)+\frac{8 N}{N+2} E(0) T_{0}^{2}+1$ which is satisfied automatically if

$$
E(0)<-\frac{(N+2) \tilde{M}\left(T_{0}\right)}{8 N T_{0}^{2}} .
$$

If the condition (22) holds then the requirement (25) also holds. Therefore, both conditions of the blow-up are satisfied at $t=T_{0}$.

Lemma 2 Redefine the constant $C_{0}$ as follows:

$$
C_{0}=\frac{4 N|E(0)|}{(N+2) \gamma^{2}}+\frac{4 \kappa}{\gamma^{2}} S_{0}(0)
$$

and keep others definitions the same as in Lemma 1. The conditions (18)-(19) are satisfied if

$$
Y(0)<\frac{8 \kappa}{\gamma} S_{0}(0)-\frac{\tilde{M}\left(T_{0, \max }\right)}{T_{0, \min }}
$$

Proof. The proof is almost identical to that for Lemma 1 except for definition of the function $\tilde{F}(t)$. Now it is defined as $\tilde{F}(t)=F(t)-Y(0) t+8 \kappa S_{0}(0) t / \gamma$. Then $\tilde{F}(t) \geq F(t)$ for any $Y(0) \leq \frac{8 \kappa}{\gamma} S_{0}(0)$. 


\section{Proof of Theorem 1}

We start with the following estimate which is obtained from (11) and from the definition of the constant $c_{3}$ :

$$
\begin{gathered}
E(t) \leq 2 \gamma \int_{0}^{t}\left(\|\nabla u\|_{2}^{2}+\|\nabla v\|_{2}^{2}+g_{1}\|u\|_{4}^{4}+g_{2}\|v\|_{4}^{4}\right) d \tau+E(0) \\
\leq \gamma c_{3} \frac{N+2}{16 N} \int_{0}^{t}\left(\|\nabla u\|_{2}^{2}+\|\nabla v\|_{2}^{2}+\|u\|_{4}^{4}+\|v\|_{4}^{4}\right) d \tau+E(0) .
\end{gathered}
$$

Rearranging the terms two in the r.h.s. of (13) as follows

$$
\begin{array}{r}
4 N E(t)+4 \int(2-N)\left(|\nabla u|^{2}+|\nabla v|^{2}\right) d x=\frac{1}{N+2}\{16 N E(t)- \\
8(N-2) \int\left(|\nabla u|^{2}+|\nabla v|^{2}\right) d x-2 N(N-2)\left[g_{1} \int|u|^{4} d x+g_{2} \int|v|^{4} d x\right]- \\
\left.4 N g(N-2) \int|u|^{2}|v|^{2} d x+8 N(N-2) \kappa \operatorname{Re} \int u \bar{v} d x\right\},
\end{array}
$$

and taking into account that

$$
16 \gamma \operatorname{Im} \int(\bar{u} x \cdot \nabla u-\bar{v} x \cdot \nabla v) d x \leq \frac{4(N-2)}{N+2}\left(\|\nabla u\|_{2}^{2}+\|\nabla v\|_{2}^{2}\right)+\frac{16 \gamma^{2}(N+2)}{N-2} X,
$$

and

$$
8 \gamma \kappa \operatorname{Im} \int|x|^{2} v \bar{u} d x \leq 4 \gamma \kappa X
$$

from (13) we obtain the following estimate:

$$
\begin{array}{r}
\frac{d^{2} X}{d t^{2}}+\frac{2(N-2)}{N+2}\left(2\|\nabla u\|_{2}^{2}+2\|\nabla v\|_{2}^{2}+N g_{1}\|u\|_{4}^{4}+N g_{2}\|v\|_{4}^{4}+2 N g \int|u|^{2}|v|^{2} d x\right) \leq \\
\leq c_{1} X+\frac{16 N}{N+2} E(t)+16 \kappa S_{0}(t)
\end{array}
$$

which implies

$$
\frac{d^{2} X}{d t^{2}}+c_{2}\left(\|\nabla u\|_{2}^{2}+\|\nabla v\|_{2}^{2}+\|u\|_{4}^{4}+\|v\|_{4}^{4}\right) \leq c_{1} X+\frac{16 N}{N+2} E(t)+16 \kappa S_{0}(t) .
$$

Then using (9) and (28) we arrive at

$$
\begin{aligned}
\frac{d^{2} X}{d t^{2}}+ & c_{2}\left(\|\nabla u\|_{2}^{2}+\|\nabla v\|_{2}^{2}+\|u\|_{4}^{4}+\|v\|_{4}^{4}\right) \leq c_{1} X+\frac{16 N}{N+2} E(0)+ \\
& +16 \kappa S_{0}(0) e^{2 \gamma t}+c_{3} \gamma \int_{0}^{t}\left(\|\nabla u\|_{2}^{2}+\|\nabla v\|_{2}^{2}+\|u\|_{4}^{4}+\|v\|_{4}^{4}\right) d \tau
\end{aligned}
$$

Next, introducing

$$
\rho(t)=\int_{0}^{t} \int_{0}^{\sigma}\left(\|\nabla u\|_{2}^{2}+\|\nabla v\|_{2}^{2}+\|u\|_{4}^{4}+\|v\|_{4}^{4}\right) d \tau d \sigma
$$


and using function $F(t)$ defined in (14), we rewrite (34) in the form

$$
\frac{d^{2}}{d t^{2}}\left(X(t)+c_{2} \rho(t)\right) \leq c_{1} X(t)+c_{3} \gamma \frac{d \rho(t)}{d t}+\frac{d^{2} F(t)}{d t^{2}} .
$$

From this inequality we obtain

$$
X(t)+c_{2} \rho(t) \leq F(t)+c_{1} \int_{0}^{t} \int_{0}^{\sigma} X(\tau) d \tau d \sigma+c_{3} \gamma \int_{0}^{t} \rho(\tau) d \tau
$$

In order to complete the proof, we use the arguments of reductio ad absurdum. Let the conditions (18)-(19) hold, but a solution of the Cauchy problem (11) nevertheless exists for all $t \in\left[0, T_{0}\right]$. Then we can define

$$
T_{1}=\sup \left\{t \in\left[0, T_{0}\right]: X(s) \leq M\left(T_{0}\right) \text { for any } s \in[0, t]\right\} .
$$

From (37) and (18) for all $t \in\left[0, T_{1}\right]$ one has

$$
\begin{array}{r}
X(t)+c_{2} \rho(t) \leq F(t)+c_{1} M\left(T_{0}\right) \frac{T_{0}^{2}}{2}+c_{3} \gamma \int_{0}^{t} \rho(\tau) d \tau \\
\leq M\left(T_{0}\right)-1+c_{1} M\left(T_{0}\right) \frac{T_{0}^{2}}{2}+c_{3} \gamma \int_{0}^{t} \rho(\tau) d \tau \\
<M\left(T_{0}\right)+c_{3} \gamma \int_{0}^{t} \rho(\tau) d \tau .
\end{array}
$$

Since $X(t) \geq 0$, by Gronwall's inequality

$$
\rho(t) \leq \frac{M\left(T_{0}\right)}{c_{2}} \exp \left(\frac{c_{3}}{c_{2}} \gamma t\right)
$$

Using this estimate back in right hand side of (39) and using function $G(t)$ defined by (16), we obtain

$$
X(t) \leq F(t)+G\left(T_{0}\right) .
$$

Therefore, using (19) we conclude that $X\left(T_{1}\right)<M\left(T_{0}\right)$ and thus $T_{1}=T_{0}$. Hence

$$
X\left(T_{0}\right) \leq F\left(T_{0}\right)+1<0,
$$

which is impossible because $X(t) \geq 0$.

\section{4 "Early-collapse"}

Now we consider the case, when the NLS equation with gain, i.e. Eq. (1a), contains attractive nonlinearity, while the nonlinearity of the system with loss i.e. Eq. (1b), as well as nonlinear coupling, are either repulsive or zero, i.e. we set

$$
g_{1}>0, \quad g_{2} \leq 0, \quad g \leq 0 .
$$


For this set of parameters, one can use the idea of the "early-collapse" suggested in 25]. The method is based on the fact that even if the energy is a growing function, its growth can be controlled and it is hence possible to choose the initial conditions $u_{0}$ and $v_{0}$ such that the blowup occurs at sufficiently early times of the evolution.

In order to obtain the growth rate of the energy, we use (11) which subject to (43) allows one to obtain

$$
\begin{aligned}
\frac{d E}{d t} \leq 2 \gamma \int\left(|\nabla u|^{2}+|\nabla v|^{2}+\kappa(\bar{u} v\right. & \left.+u \bar{v})-\frac{g_{1}}{2}|u|^{4}-\frac{g_{2}}{2}|v|^{4}-g|u|^{2}|v|^{2}\right) d x+ \\
& +2 \gamma \kappa\left(\|u\|_{2}^{2}+\|v\|_{2}^{2}\right) \leq 2 \gamma E(t)+2 \kappa \gamma S_{0}(t) .
\end{aligned}
$$

Thus using (9) and the definition of the energy (10) we derive

$$
E(t) \leq\left(E(0)+2 \kappa \gamma S_{0}(0) t\right) e^{2 \gamma t}=: E_{\max }(t)
$$

Next, we derive the following estimate

$$
16 \gamma \operatorname{Im} \int(\bar{u} x \cdot \nabla u-\bar{v} x \cdot \nabla v) d x \leq \frac{16 \gamma^{2}}{N-2} X+4(N-2)\left(\|\nabla u\|^{2}+\|\nabla v\|^{2}\right),
$$

and from the identity (13) we obtain

$$
\frac{d^{2} X}{d t^{2}} \leq c_{4}^{2} X+4 N E_{\max }(t)+4 \kappa N S_{0}(t), \quad c_{4}=2 \gamma \sqrt{\frac{\kappa}{\gamma}+\frac{N+2}{N-2}} .
$$

Now one can obtain the upper bound for $X$ :

$$
X(t) \leq Z(t) e^{c_{4} t},
$$

where

$$
Z(t):=X(0)+\int_{0}^{t} e^{-2 c_{4} s}\left[Y(0)-c_{4} X(0)+4 N \int_{0}^{s} e^{c_{4} \sigma}\left[E_{\max }(\sigma)+\kappa S_{0}(0) e^{2 \gamma \sigma}\right] d \sigma\right] d s,
$$

and the function $E_{\max }(\sigma)$ was defined in (45)).

The obtained result can be reformulated as the following Theorem.

Theorem 2 Let $N \geq 3$, the coefficients $g_{1}, g_{2}$ and $g$ satisfy 43), and initial conditions $u_{0}$ and $v_{0}$ in the Cauchy problem (1) are such that the function $Z(t)$ defined by (49) has a real positive zero $T_{*}$. Then the solution of the problem (1) does not exist in the interval $t \in\left[0, T_{*}\right]$.

Since the occurrence of the blowup is now reduced to the study of the zeros of $Z(t)$ and this function depends on several parameters of the problem, we limit further consideration of this section by the numerical analysis. To this end now, as well as in all other numerical examples below, we limit the analysis to the Gaussian initial conditions (as the most typical for experimental settings):

$$
u_{0}(x)=\frac{A}{\pi^{N / 4} a^{N / 2}} \exp \left(-\frac{|x|^{2}}{2 a^{2}}\right), \quad v_{0}(x)=\frac{B}{\pi^{N / 4} b^{N / 2}} \exp \left(-\frac{|x|^{2}}{2 b^{2}}\right)
$$



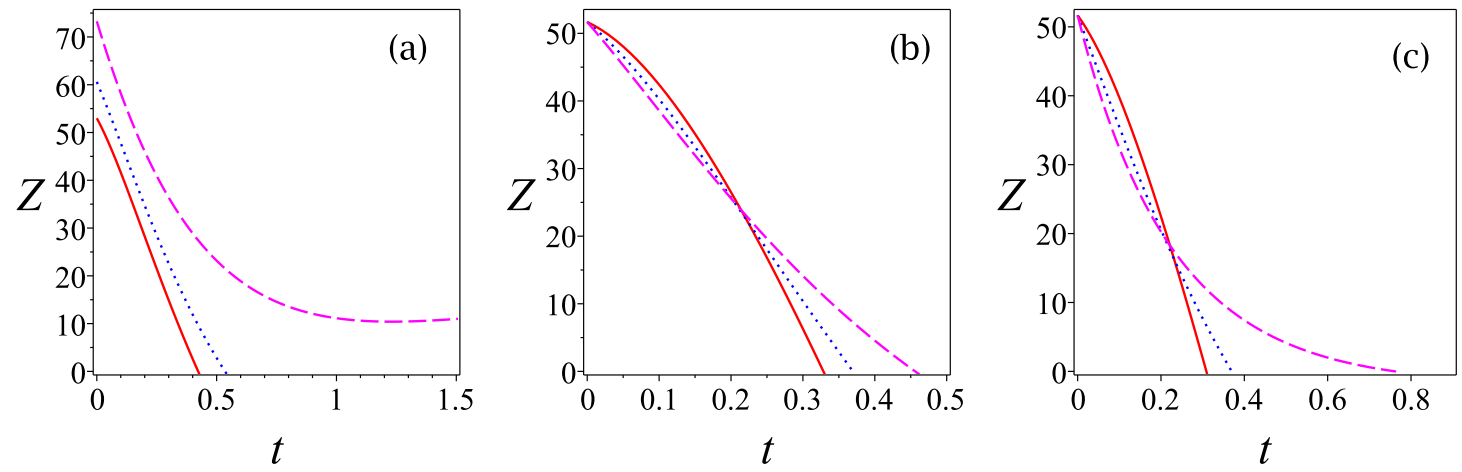

Figure 1: Plots of $Z(t)$ for $N=3, g_{1}=4, g_{2}=-1, g=-0.5$, and the Gaussian initial conditions (50) with $A=5.8$ and $a=b=1$. The other parameters are as follows. Panel (a): $\gamma=0.5, \kappa=1$, $B=1.3$ (solid line), $B=2.6$ (dotted line), $B=3.9$ (dashed line). Panel (b) $\gamma=0.5, B=0.9$, $\gamma=0.15$ (solid line), $\gamma=0.3$ (dotted line), $\gamma=0.45$ (dashed line). Panel (c): $\gamma=0.5, B=0.9$, $\kappa=0.4$, (solid line), $\kappa=0.8$ (dotted line), $\kappa=1.2$ (dashed line).

where $a, b, A$, and $B$ are positive constants.

In Fig. 1 we show plots of the function $Z(t)$ for several particular choices of the parameters in the case $N=3$, where we observe that for a proper choice of the initial conditions and the system parameters zeros of the function $Z(t)$ indeed exist. The time where $Z(t)$ becomes zero gives the respective $T_{*}$ (with is the minimal root obtained numerically). In Fig. 1(a) we show the behavior of $Z(t)$ for different values $B$, which corresponds to different initial conditions $v_{0}(x)$ for the equation (1b) with dissipation, provided the input of the NLS with gain, i.e. $u_{0}(x)$ is fixed. Increase of the initial amplitude $B$ of the pulse subjected dissipation [i.e $v_{0}(x)$ ] results in larger values $T_{*}$ and eventually leads to nonexistence of the zeros of $Z(t)$. In the cases where $T_{*}$ does not exist, the question about the finite time blowup remains open.

In Fig. 1(b) we address different values of the gain/loss coefficient $\gamma$, and in Fig. 1(c) we illustrate different values of the coupling coefficient $\kappa$ at fixed initial conditions. We observe increase of $T_{*}$ for stronger gain and dissipation [panel (b)], as well as for stronger coupling [panel (c)].

\section{A remark on the Manakov model}

The sufficient blowup conditions formulated in Sec. 2 allow for a simplification in the case of equal nonlinear coefficients: $g_{1}=g_{2}=g>0$ (below we refer to this model as Manakov's one, after the original work [26]). Without loss of generality now we can set $g_{1}=g_{2}=g=1$ (which can be achieved by the renormalization $\sqrt{g} u \rightarrow u, \sqrt{g} v \rightarrow v)$.

An interesting property of the Manakov model is the existence of (at least) two integrals of motion. Indeed, straightforward computations show that the quantities

$$
S_{1}(t)=S_{1}(0) \quad \text { and } \quad S(t):=\kappa S_{0}(t)-\gamma S_{2}(t)=\kappa S_{0}(0)-\gamma S_{2}(0)
$$

do not depend on time. Then, from (8) one obtains

$$
\frac{d^{2} S_{0}}{d t^{2}}+4 \omega^{2} S_{0}=4 \kappa S
$$


where $\omega=\sqrt{\kappa^{2}-\gamma^{2}}$ as defined in (5). Thus in the case of unbroken the $\mathcal{P} \mathcal{T}$-symmetric phase (2) the quantity $S_{0}(t)$ undergoes oscillatory dynamics:

$$
\begin{array}{r}
S_{0}(t)=\frac{\kappa S}{\omega^{2}}+S_{01} \cos (2 \omega t)+S_{02} \sin (2 \omega t), \\
S_{01}=S_{0}(0)\left(1-\frac{\kappa}{\omega^{2}}\right)+S_{2}(0) \frac{\gamma \kappa}{\omega^{2}}, \quad S_{02}=S_{3}(0) \frac{\gamma}{\omega},
\end{array}
$$

and hence there exists an upper bound for $S_{0}(t)$ [c.f. (9)]:

$$
S_{0}(t) \leq S_{0, \max }:=\frac{\kappa S}{\omega^{2}}+\sqrt{S_{01}^{2}+S_{02}^{2}}
$$

In order to obtain sufficient conditions of the blowup in the Manakov case, we again employ Eq. (13) and Eq. (29) and, using that the quantity $S_{1}(t)$ is conserved, we can replace the estimate (32) by the following one:

$$
\begin{array}{r}
\frac{d^{2} X}{d t^{2}}+\frac{2(N-2)}{N+2}\left(2\|\nabla u\|_{2}^{2}+2\|\nabla v\|_{2}^{2}+N g_{1}\|u\|_{4}^{4}+N g_{2}\|v\|_{4}^{4}+2 N g \int|u|^{2}|v|^{2} d x\right) \leq \\
\leq c_{1} X+\frac{16 N}{N+2}\left(E(t)-\kappa S_{1}(0)\right) .
\end{array}
$$

One can repeat all the subsequent steps of the proof of Theorem 1 using the newly obtained estimate (56). Therefore, in the Manakov case Theorem 1 can be reformulated with functions $F$, $M$ and $G$ replaced by $\hat{F}, \hat{M}$ and $\hat{G}$, respectively, where the new functions are defined as

$$
\begin{aligned}
& \hat{F}(t)=X(0)+Y(0) t+\frac{8 N}{N+2}\left(E(0)-\kappa S_{1}(0)\right) t^{2} \\
& \hat{M}(t)=\sup _{\tau \in[0, t]} \hat{F}(\tau)+1 \\
& \hat{G}(t)=\hat{M}(t)\left(c_{1} \frac{t^{2}}{2}+\exp \left(\frac{48 N \gamma t}{N+2}\right)-1\right) .
\end{aligned}
$$

\section{$6 \quad$ Numerical illustrations}

The analytical results obtained above give sufficient conditions for the finite time blowup, but do not describe the blowup dynamics and its dependence on the parameters of the model, i.e. $\gamma, \kappa$, $g_{1}, g_{2}$ and $g$. In order to understand better the effect of those parameters on the phenomenon of blowup, now we resort to numerical analysis of the Cauchy problem (11). We concentrate on the case $N=3$ which is the most interesting one from the physical point of view. We also set $g_{1}=g_{2}$ and consider how the blowup depends on the nonlinear coupling $g$ and on the gain/loss coefficient $\gamma$. As we mentioned above, without loss of generality we can set $g_{1}=g_{2}=1$ (which is achieved by the renormalization $\sqrt{g_{1}} u \rightarrow u, \sqrt{g_{2}} v \rightarrow v, g / g_{1}=g / g_{2} \rightarrow g$ ). Using another evident renormalization, without loss of generality we can set $\kappa=1$. Therefore we concentrate our attention on the system

$$
\begin{aligned}
& i u_{t}=-\Delta u+i \gamma u+v-\left(|u|^{2}+g|v|^{2}\right) u \\
& i v_{t}=-\Delta v-i \gamma v+u-\left(g|u|^{2}+|v|^{2}\right) v
\end{aligned}
$$


where $x \in \mathbb{R}^{3}$ and $t \geq 0$.

We first notice that the blowup conditions (18)-(19) can be easily satisfied by a proper choice of the initial pulses $u_{0}$ and $v_{0}$. Several examples are presented in Fig. 2 which shows spatial profiles of initial conditions $u_{0}(x)$ and $v_{0}(x)$ chosen in the form of the Gaussian beams (50) and the behavior of the associated functions $F(t)$ and $G(t)$ defined by (14) and (16). Panels Fig. 2 (ab) correspond to a situation when the blowup conditions are not satisfied as functions $F(t)$ and $G(t)$ grow monotonously. However, increasing powers $A$ and $B$ of the input beams [or one of the beams, see Fig. 2 (c-d)] or decreasing the characteristic widths $a$ and $b$ of the beams [Fig. 2(e-f)], one can easily satisfy the blowup conditions.

We also performed numerical simulations of the three-dimensional system (60) in the spherical symmetric case, i.e. assuming that the functions $u$ and $v$ depend only on the radius $r=|x|$ in the spherical coordinates and do not depend on the polar and azimuthal angles. In this case it is convenient to introduce new functions $p(r, t)=r u$ and $q(r, t)=r v$ and to reformulate the problem as follows

$$
\begin{aligned}
& i p_{t}=-p_{r r}+i \gamma p+q-r^{-2}\left(|p|^{2}+g|q|^{2}\right) p, \\
& i q_{t}=-q_{r r}-i \gamma q+p-r^{-2}\left(g|p|^{2}+|q|^{2}\right) q .
\end{aligned}
$$

We also limited the numerical study to a finite interval $r \in[0, L]$ subject to the zero boundary conditions $p(0, t)=q(0, t)=p(L, t)=q(L, t)=0$. The interval width $L$ is taken sufficiently large such that increase of $L$ practically does not affect the results presented below.

We solved system (61) using a semi-implicit finite-difference scheme proposed in [27. The numerical simulations were carried on up to the time $t_{*}$ when the ratios $\left|u\left(t_{*}, 0\right)\right| /\left|u_{0}(0)\right|$ and $\left|v\left(t_{*}, 0\right)\right| /\left|v_{0}(0)\right|$ were of order $10^{2}$, and then the simulations were interrupted. We used the spatial step of order $10^{-5}$ and the adaptively decreasing temporal step which was of order $10^{-5}$ in the beginning of simulations (i.e. at $t=0$ ) and of order $10^{-7}$ at the time $t=t_{*}$ of the termination of the simulations. We also performed several additional runs with smaller spatial steps and checked convergence of the numerical solution.

In the numerical simulations we have observed different scenarios of the dynamics: dispersion of the initial pulses, growth of the solution and its derivative at the origin $x=0$ in one of the components (either with gain or with dissipation), and the simultaneous growth in both the components. The dispersion corresponds to the spreading of the initial pulses (which eventually occupy the entire computational domain $[0, L]$ ), while the observed growth is a presumable manifestation of the blowup. Our numerical results allow us to conjecture that depending on the initial conditions and parameters of the model the blowup can occur either in both or in only one component. In the latter case this can be a component either with gain or with damping.

Summarizing results of dynamical simulation of system, we first of all conjecture that the obtained above blowup conditions (18)-(19) are not necessary for the blowup to occur: it is easy to find initial conditions that do not satisfy (18)-(19) but nevertheless grow in numerical simulations. Moreover, our numerics indicate that the growth can occur even for initial conditions with $E(0) \geq 0$ and $Y(0) \geq 0$, i.e. in the case when conditions (18)-(19) obviously can not be satisfied for any $T_{0}$.

Several (the most interesting) examples of the blowup-like dynamics obtained from numerical solution of system (61) are presented in Fig. 3. The case of the unbroken $\mathcal{P} \mathcal{T}$ symmetry is illustrated in Fig. 3(a) from where one observes that positive and negative values of $g$ result in different scenarios of the blowup-like dynamics. Namely, for $g=1$ (this is the attractive interspecies nonlinearity) we observe that the the intensity growth occurs in both the components. 

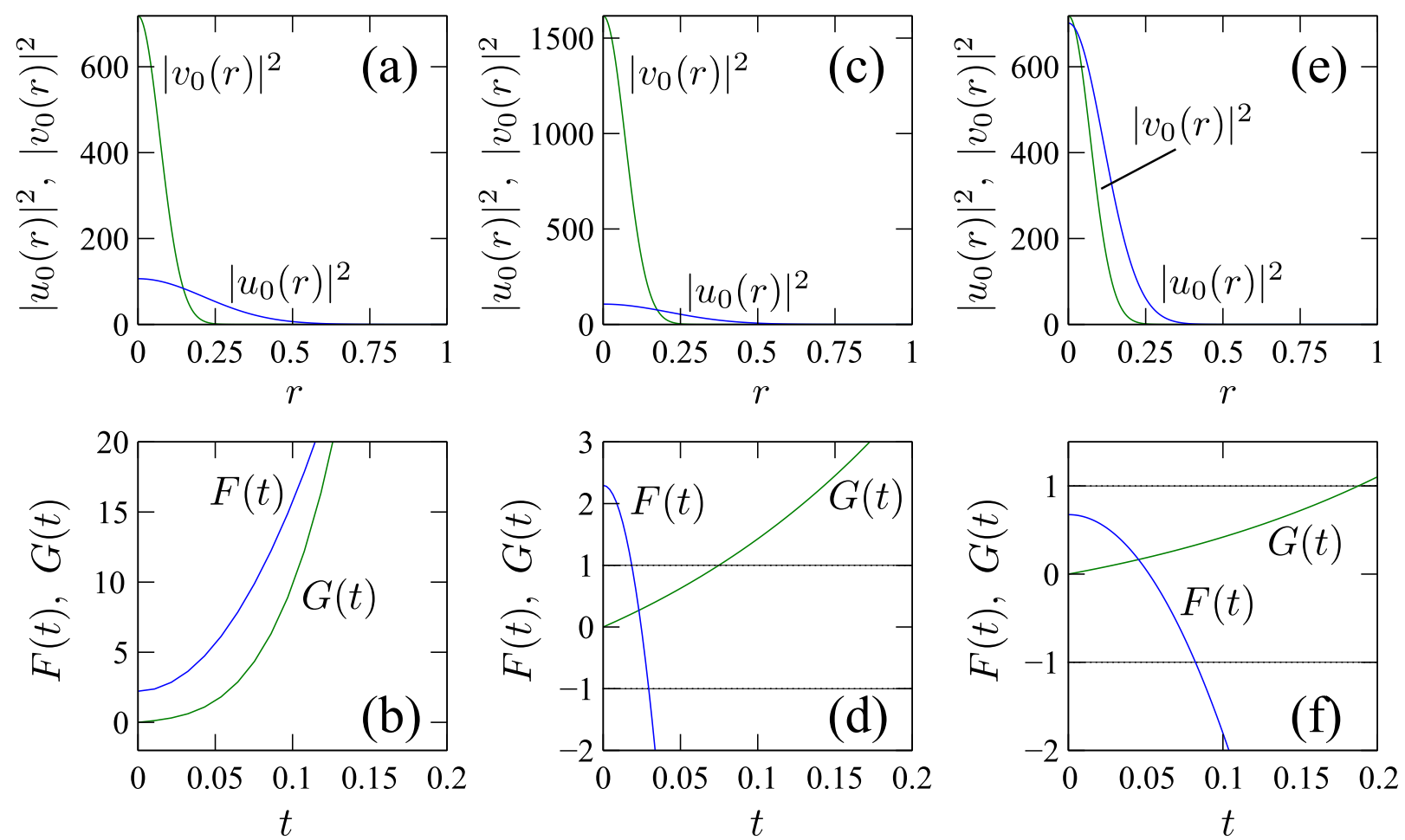

Figure 2: (a) and (b) Plots of initial conditions (50) for $N=3, A=4, B=2, a=0.3$ and $b=0.1$ (with $r=|x|$ ) and behavior of the associated functions $F(t)$ and $G(t)$ defined by (14) and (16) with $\kappa=1, \gamma=0.5, g_{1}=g_{2}=1$ and $g=-0.5$; (c) and (d) show the same as (a) and (b) but with $B=3$; (e) and (f) show the same as (a) and (b) but with $b=0.16$. Notice that the blowup conditions (18)-(19) are not satisfied in (a)-(b), but become satisfied in (c)-(d) and (e)-(f). 

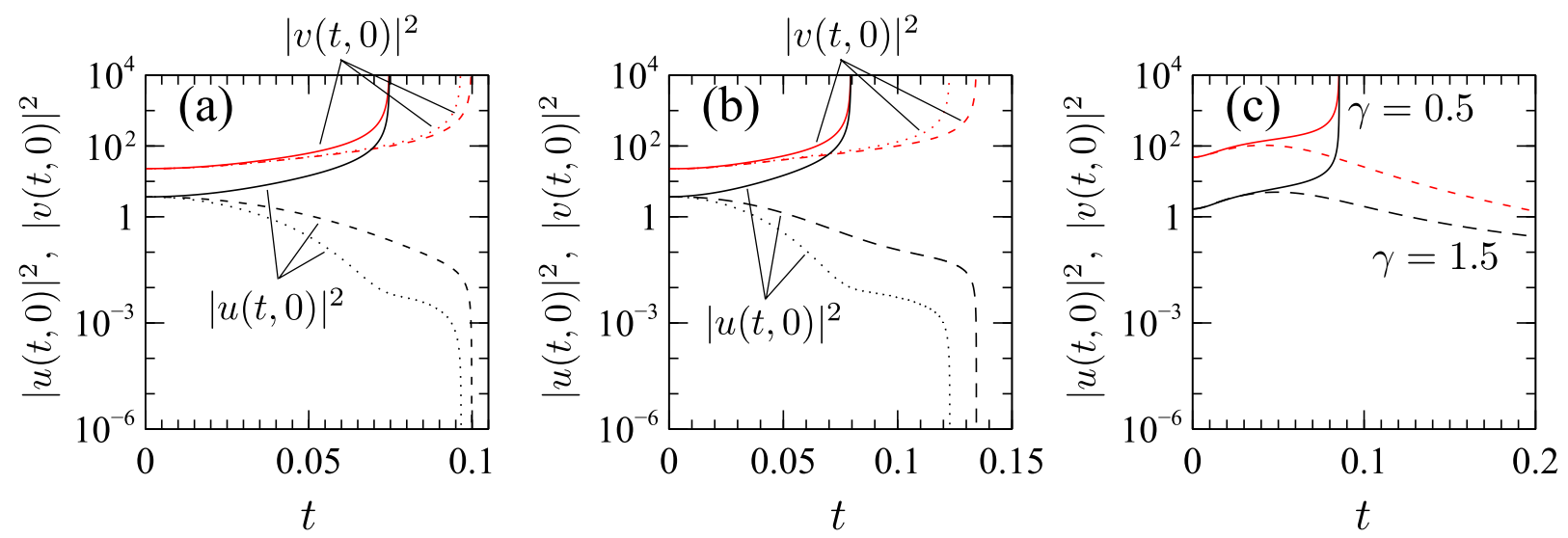

Figure 3: (a)-(b) Numerically obtained dynamics with the initial conditions (50) with $A=4.5$, $B=4, a=1$ and $b=0.5$ for $\gamma=0.5$ (a) and $\gamma=1.5$ (b). Solid, dashed and dotted lines correspond to $g=1, g=-1$ and $g=-2$, respectively. (c) The dynamics obtained for $A=0.5$, $B=2.7, a=b=0.3$ and $g=1$. The solution blows up for the unbroken $\mathcal{P} \mathcal{T}$ symmetry $(\gamma=0.5$, solid curves) but disperses (at least, at the initial stage of the evolution) for the broken $\mathcal{P} \mathcal{T}$ symmetry $(\gamma=1.5$, dashed lines). The dependencies are shown in the semi-logarithmic scale.

However for $g=-1$ and $g=-2$ the same initial conditions display growth only in the dissipative component $v(x, t)$ while in the gain component $u(x, t)$ the intensity at the origin decays. Notice also that the maximal squared amplitude in the component with gain $\max _{r \in[0, L]}|u(x, t)|^{2}$ for $g=-1$ and $g=-2$ (not shown on the plot) was of the order of 1 at the moment when the simulations were terminated. It is also interesting to notice that for $g=-1$ and $g=-2$ the only component "responsible" for the blowup is the one with dissipation [i.e. $v(x, t)$ ] and that the growth with $g=-2$ (the dotted curves) occurs earlier than that with $g=-1$ (the dashed curves) in spite of the additional "defocusing" that large negative values of $g$ induce. We finally notice that the case $g=-2$ is not covered by the condition of the Theorem 1 , which is another illustration for the fact that the obtained blowup conditions (18)-(19) are sufficient but not necessary.

Repeating the same simulations for $\gamma=1.5$ [the broken $\mathcal{P} \mathcal{T}$ symmetry, Fig. 3 (b)] we do not observe any quantitative difference in the blowup scenarios with respect to the case $\gamma=0.5$. However it is interesting to notice that for the chosen initial conditions and for the three different values of $g$ addressed in Fig. 3(b) the numerical blowup for the broken $\mathcal{P} \mathcal{T}$ symmetry occurs later than that with the unbroken $\mathcal{P} \mathcal{T}$ symmetry in Fig. 3 (a) (this is especially well-visible for $g=-1$ and $g=-2$ ). However this behavior is conditioned by the chosen initial profiles. It is also possible to find initial conditions for which the broken $\mathcal{P} \mathcal{T}$ symmetry precipitates the intensity growth (with respect to the unbroken $\mathcal{P} \mathcal{T}$ symmetry). Another interesting effect is that it is possible to select the initial conditions which grow for the unbroken $\mathcal{P} \mathcal{T}$ symmetry but disperse (at least, at the initial stage of the evolution) for the broken $\mathcal{P} \mathcal{T}$ symmetry [Fig. 3(c)], which is an indication of absence of any direct relation between the blowup phenomenon and the $\mathcal{P} \mathcal{T}$ symmetry breaking.

In Fig. 4 we demonstrate how increase of the amplitude $A$ of the initial conditions [Fig. 4(a)] and increase of the parameter of the $\mathcal{P} \mathcal{T}$ symmetry $\gamma$ [Fig. 4 (b)] changes the dynamics from the dispersive (and presumably global) one to the blowup. 

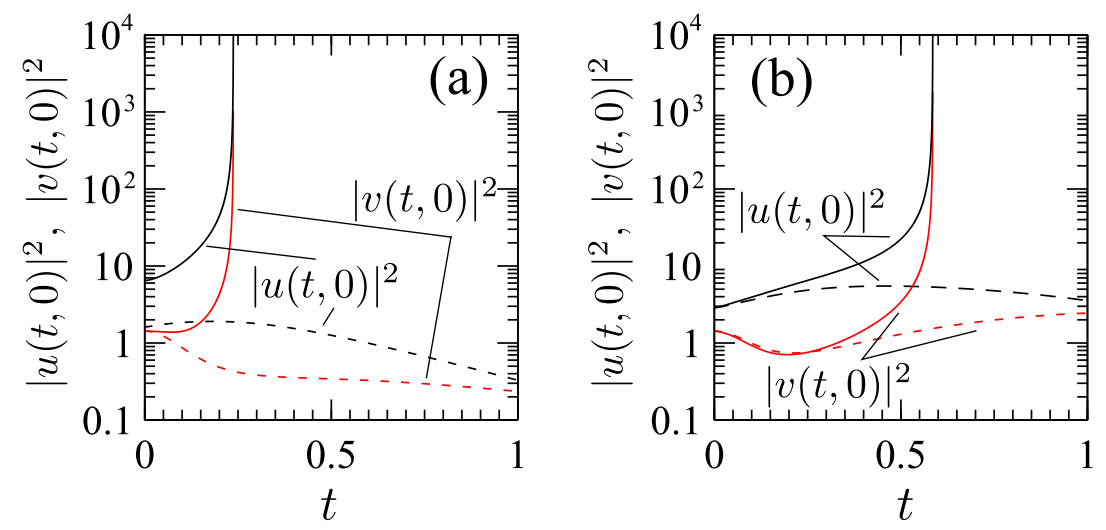

Figure 4: Change of the dynamics after modification of parameter $A$ of the initial conditions [panel (a)] and parameter of $\mathcal{P} \mathcal{T}$ symmetry $\gamma$ [panel (b)]. The dynamics in (a) is obtained for the initial conditions (50) with $B=1, a=1$ and $b=0.5, A=3$ (dashed curves) and $A=6$ (solid curves), and with $\gamma=0.5$. The dynamics in (b) is obtained for the initial conditions (50) with $A=B=1, a=1, b=0.5$, and with $\gamma=0.5$ (dashed curves) and $\gamma=0.9$ (solid curves). In both panels $g=1$. The dependencies are shown in the semi-logarithmic scale.

\section{Concluding remarks}

In this paper we have established sufficient conditions for finite-time blow up of initial pulses whose evolution is described by the coupled $\mathcal{P} \mathcal{T}$-symmetric NLS equations, one of which has linear gain and another one has dissipation. The equations include both linear and nonlinear coupling. The proofs were presented for the two cases. In the first situation the both NLS equations have attractive (focusing) self-phase nonlinearities and either attractive or weakly repulsive cross-phase nonlinearities (i.e. nonlinear coupling coefficients). In the second case the equation with gain was self-attracting while all other nonlinear interactions were repulsive (defocusing).

Neither proof nor the results rely on whether the $\mathcal{P} \mathcal{T}$ symmetric phase is broken or not. In the formulations of theorems the gain/dissipation strength and the coupling enter as independent parameters (unlike this happens in the linear problem, where their relation is the only significant parameter). This is however expectable in view of the fact that the blowup is an essentially nonlinear phenomenon.

We also performed numerical studies of the initial stages of evolution of the initial data. The consideration was limited to the simplest Gaussian shapes; comprehensive numerical study of the initial data is left as an open question. However even these first studies revealed interesting counter-intuitive dynamics like dispersion of pulses in the case of broken (linear) symmetry and blow up of the same pules in the case of unbroken symmetry, or occurrence of collapse of the field in the equation with dissipation with the field subjected to gain reaming bounded.

Finally, we notice that the developed theory does not allow for direct generalization for the critical collapse, which is left, so far, as another open problem. 


\section{Acknowledgments}

JPD and MF acknowledge support of the FCT (Portugal) grant PEst-OE/MAT/UI0209/2013.

VVK and DAZ acknowledge support of the FCT (Portugal) grants PEst-OE/FIS/UI0618/2014 and PTDC/FIS-OPT/1918/2012.

\section{References}

[1] C. M. Bender and S. Boettcher. Real spectra in mon-Hermitian Hamiltonians having PT symmetry, Phys. Rev. Lett. 80 : 5243-5246 (1998).

[2] C. M. Bender. Making sense of non-Hermitian Hamiltonians, Rep. Prog. Phys. 70 : 947 (2007).

[3] A. Ruschhaupt, F. Delgado, and J. G. Muga. Physical realization of PT-symmetric potential scattering in a planar slab waveguide, J. Phys. A 38 : L171-L176 (2005).

[4] R. El-Ganainy, K. G. Makris, D. N. Christodoulides, and Z. H. Musslimani. Theory of coupled optical PT-symmetric structures, Opt. Lett. 32 : 2632 (2007).

[5] A. Guo, G. J. Salamo, M. Volatier-Ravat, V. Aimez, G. Siviloglou, and D. N. Christodoulides, Observation of PT-symmetry breaking in complex optical potentials. Phys. Rev. Lett. 103 : 093902 (2009).

[6] C. E. Rüter, K. G. Makris, R. El-Ganainy, D. N. Christodoulides, M. Segev, and D. KIP. Observation of parity-time symmetry in optics, Nat. Phys. 6 : 192 (2010).

[7] Z. H. Musslimani, K. G. Makris, R. El-Ganainy, and D. N. Christodoulides. Optical solitons in PT periodic potentials. Phys. Rev. Lett. 100 : 030402 (2008)

[8] R. Driben and B. A. Malomed. Stability of solitons in parity-time-symmetric couplers, Opt. Lett. $36: 4323$ (2011).

[9] N. V. Alexeeva, I. V. Barashenkov, A. A. Sukhorukov, and Y. S. Kivshar. Optical solitons in PT-symmetric nonlinear couplers with gain and loss, Phys. Rev. A 85: 063837 (2012).

[10] Y. V. Bludov, V. V. Konotop, and B. A. Malomed. Stable dark solitons in PT-symmetric dual-core waveguides, Phys. Rev. A 87 : 013816 (2013).

[11] I. V. Barashenkov, S. V. Suchkov, A. A. Sukhorukov, S. V. Dmitriev, and Y. S. Kivshar. Breathers in PT-symmetric optical couplers, Phys. Rev. A 86 : 053809 (2012).

[12] Y. V. Bludov, R. Driben, V. V. Konotop, and B. A. Malomed. Instabilities, solitons and rogue waves in PT-coupled nonlinear waveguides, J. Opt. 15 : 064010 (2013).

[13] H. Cartarius and G. Wunner. Model of a PT-symmetric Bose-Einstein condensate in a $\delta$-function double-well potential, Phys. Rev. A 86 : 013612 (2012).

[14] M. Kreibich, J. Main, H. Cartarius, and G. Wunner. Hermitian four-well potential as a realization of a PT-symmetric system, Phys. Rev. A 87 : 051601(R) (2013). 
[15] Y. V. Kartashov, V. V. Konotop, and D. A. Zezyulin. CPT-symmetric spin-orbitcoupled condensate (submitted).

[16] D. C. Roberts, and A. C. Newell. Finite-time collapse of $N$ classical fields described by coupled nonlinear Schrödinger equations, Phys. Rev. E 74 : 047602 (2006).

[17] V. Prytula, V. Vekslerchik and V. PÉrez-García. Collapse in coupled nonlinear Schrödinger equations: Sufficient conditions and applications, Physica D 238 : 1462-1467 (2009).

[18] A. JÜngel and R.-M. WeIshäUpl. Blow-up in two-component nonlinear Schrödinger systems with an external driven field, Math. Mod. Meth. App. Sci. 23 : 1699-1727 (2013).

[19] L. Bergé. Wave collapse in physics: principles and applications to light and plasma waves, Phys. Rep. 303 : 259-370 (1998).

[20] M. Ohta and G. Todorova. Remarks on global existence and blowup for damped nonlinear Schrödinger equations, Disc. Cont. Dyn. Syst. 23 : 1313-1325 (2009).

[21] M. Tsutsumi. Nonexistence of global solutions to the Cauchy problem for the damped nonlinear Schrödinger equations, SIAM J. Math. Anal. 15 : 317-366 (1984).

[22] J.-P. Dias and M. Figueira. On the blowup of solutions of a Schrödinger equation with an inhomogeneous damping coefficient, Comm. Contemp. Math. 16 : 1350036-1350046 (2014).

[23] T. Kato, Perturbation Theory for Linear Operators, Berlin, Springer, 1980.

[24] C. Sulem and P.-L. Sulem, The Nonlinear Schrödinger Equation, Springer, New York, 1999.

[25] V. V. Konotop and P. Pacciani. Collapse of solutions of the nonlinear Schrödinger equation with a time-dependent nonlinearity: Application to Bose-Einstein condensates, Phys. Rev. Lett. $94: 240405$ (2005).

[26] S. V. Manakov. On the theory of two-dimensional stationary self-focusing of electromagnetic waves, Zh. Eksp. Teor. Fiz. 65 : 505 (1973) [Sov. Phys. JETP 38 : 248 (1974)].

[27] V. A. Trofimov and N. V. Peskov. Comparison of finite-difference schemes for the GrossPitaevskii equation, Math. Model. Anal. 14 : 109-126 (2009). 